\title{
What case reports can and cannot reveal
}

\author{
David F. Albertini ${ }^{1}$
}

Published online: 26 September 2015

(C) Springer Science+Business Media New York 2015

Among the discussions that publishers and editors engage in without fail are the metrics of success for the traditional and new wave "journals" where discovery is delivered to the thirsty consumer, most of whom now prefer the click of a mouse to the touch of papyrus! One of the first discussions of this kind I recall had much to do with what kind of articles enjoy the "hit parade" and what type slip through the keyboard fated to the great hard disk in the sky — or maybe the molten core of our planet. Among this latter category of publications would be the case report. JARG welcomes the occasional case report that offers our readership a morsel to mull over, and we encourage authors of such submissions to use this as an opportunity to survey what is often a narrow or neglected field that might be deserving of a rebirth. With this backdrop, we feature three case reports this month that take us through past, present, and future dimensions of human ARTs.

The first of these, from Itoi et al., is emblematic of a stream of studies indicating that many of the characteristics exhibited by human embryos that were believed indicative of poor quality are simply not the clearcut discriminators we had once thought (Birth of nine normal healthy babies following transfer of blastocysts derived from human single-pronucleate zygotes 10.1007/s10815-015-0518-y). The debate over how well our predetermined biomarkers of malaise fulfill forecasting criteria for development to blastocysts lingers on,

Capsule With the ever-expanding production of data spawned by the wave of technological breakthroughs, is it no wonder that the simple observational facts get overlooked in what is supposed to be a time of enlightenment in human ARTs.

David F. Albertini

DALBERTINI@kumc.edu

1 University of Kansas Medical Center, Kansas, KS, USA and these findings reinforce trends to extend culture before making choices for embryo transfer or cryopreservation. But the fact that gross deviations in pronuclear appearance and transition kinetics through the first cell cycle continue to be accessible targets for machine-based (vs hominin-based) learning tools in embryo selection strategies adds an ironic twist to the community of term gestation prognosticator "wannabees." Poor grades in primary school do not necessarily reflect adult competencies and achievements.

The second case report is by Aghajanova et al., and takes as a point of departure the power of preimplantation genetic screening (PGS) for treating a male patient with non-mosaic Down syndrome (Birth of a healthy child after preimplantation genetic screening of embryos from sperm of a man with non-mosaic Down syndrome 10.1007/s10815-015-0525-z). Here, the tables are turned as the obvious indication for PGS results in a favorable outcome, extending the purposeful menu of options patients have access to. Moreover, digging deeper into their observations from trophectoderm biopsies, two attempts gave definitive euploid results for 12 out of 13 of the original blastocysts and no signs of trisomy 21 . As the authors point out, their case is consistent with results from previous reports of non-mosaic males having healthy children and re-emphasizes the growing belief that rectifying chromosome balance in gametes is a more likely outcome from spermatogenesis than oogenesis, where so many aneuploidies can traverse gamete maturation and find their way into the zygotic genome. This report also takes our readership into several larger questions having to do with the ethical and societal issues raised by ART-enabled children of Down syndrome individuals.

Last but not least, we return to the topic of fertilization and what lies beneath the many cases of failure where the search for a culprit - male or female - is not so obvious. As covered recently in JARG and elsewhere, that guilt by association be 
assigned to sperm lacking phospholipase C-zeta (PLCz) is to be expected, and typically defects in enzyme activity would be associated with poor sperm/semen quality. The case report by Chithiwala et al. is unique in demonstrating persistence in problem solving that finally paid off for their patients (Phospholipase C-zeta deficiency as a cause for repetitive oocyte fertilization failure during ovarian stimulation for in vitro fertilization with ICSI: a case report 10.1007/s10815-0150531-1). With apparently normal semen, the many attempts to achieve pregnancy for this couple were repeatedly thwarted by an inability to fertilize. Careful evaluation uncovered a deficiency in PLCz as the likely cause and moved management directly into ICSI with ionophoremediated egg activation. Their trials and tribulations are worth taking note of, but in the end, success came from an unlikely turn of events. The two blastocysts that were finally obtained and transferred resulting in a live birth came not from treatment of normal MII oocytes but from in vitro matured ones! So after many reports downplaying the clinical utility of in vitro matured oocytes, at least in this isolated case that begged for severe interventions designed to compensate for defective sperm, one again has to wonder what went right for this couple who took home a baby.

Case reports of the sort noted here all bring to our ART platform for discussion both surprises and concerns. By definition, the limited sample size most always connotes the sense of a "blip-on-the-radar-screen." Despite this limitation, the surprises often carry with them incentives for further exploration that may or may not require invoking the latest technocratic embellishments peppering the world of contemporary ARTs. Simple observations can go a long way if we adhere to the following quote from Yogi Berra, a former New York Yankee catcher:

"You can see a lot if you look!" 